\title{
Answer to the Letter to the Editor of M. Ganau et al. concerning "Osteoporotic thoracolumbar compression fractures: long-term retrospective comparison between vertebroplasty and conservative treatment" by K. Martikos et al. [Eur Spine J (2018) doi: 10.1007/s00586-018-5605-1]
}

\author{
Konstantinos Martikos ${ }^{1}$
}

Received: 5 May 2018 / Accepted: 20 May 2018 / Published online: 6 June 2018

(c) Springer-Verlag GmbH Germany, part of Springer Nature 2018

We would like to thank the readers for the interest in our manuscript and answer their questions as follows:

Regarding choice of treatment, each patient was evaluated individually considering general health and the need for early ambulation. All patients were informed of both treatments, and final decision was made considering both patients' preference and general health conditions.

In all cases, initial intention for VP was unilateral; therefore, initial approach was always unilateral. When PMMA diffusion was not symmetrical within the vertebral body, a contralateral approach was performed during the same surgical procedure in order to obtain a more symmetric augmentation. The objective of our study takes into consideration mainly long-term outcomes; the only early postoperative data that were collected were PMMA complications. Therefore, our study does not intend to argue with early beneficial effects of PV.
Regarding our data that sustain a higher long-term adjacent fracture rate in patients treated with PV, we admit that in every study there may be numerous statistical methods for a more detailed analysis, but on the other hand there are numerous studies reporting higher adjacent fracture rate in patients treated with PV.

Regarding future fracture prevention, we fully agree that medical treatment for osteoporosis and appropriate lifestyle are very important; therefore, all patients admitted in our Institute with osteoporotic fractures receive appropriate evaluation for adequate treatment with anti-osteoporotic drugs after orthopedic treatment (CT or PV that may be).

\section{Compliance with ethical standards}

Conflict of interest No conflict of interest.
Konstantinos Martikos

kostasmartikos@gmail.com

1 Spinal Deformities Division, Istituto Ortopedico Rizzoli, Via Pupilli 1, 40136 Bologna, Italy 